\title{
A validated computational framework to evaluate the stiffness of 3D printed ankle foot orthoses
}

\author{
Alessio lelapi ${ }^{a, b}$, Nicolas Lammens ${ }^{c, d}$, Wim Van Paepegem ${ }^{d}$, Malcolm Forward ${ }^{e}$, Jan Patrick Deckers ${ }^{f}$, \\ Miguel Vermandel ${ }^{f}$ and Matthieu De Beule ${ }^{a}$ \\ ${ }^{a}$ Department of Electronics and Information Systems, Institute Biomedical Technology (IBiTech) - bioMMeda, Ghent University, Gent,

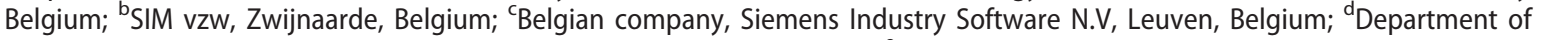 \\ Materials Science \& Engineering, Ghent University, Zwijnaarde, Belgium; ${ }^{\mathrm{e}}$ Gait \& Movement Analysis Laboratory - Cerebral Palsy \\ Reference Centrum, University Hospital Ghent, Gent, Belgium; 'Belgian company, V!GO NV, Wetteren, Belgium
}

\begin{abstract}
The purpose of this study was to create and validate a standardized framework for the evaluation of the ankle stiffness of two designs of 3D printed ankle foot orthoses (AFOs). The creation of four finite element (FE) models allowed patient-specific quantification of the stiffness and stress distribution over their specific range of motion during the second rocker of the gait. Validation was performed by comparing the model outputs with the results obtained from a dedicated experimental setup, which showed an overall good agreement with a maximum relative error of $10.38 \%$ in plantarflexion and $10.66 \%$ in dorsiflexion. The combination of advanced computer modelling algorithms and 3D printing techniques clearly shows potential to further improve the manufacturing process of AFOs.
\end{abstract}

\section{ARTICLE HISTORY}

Received 7 June 2018

Accepted 27 March 2019

\section{KEYWORDS}

Finite element models; experimental setup; ankle foot orthosis (AFO); stiffness; gait

\section{Introduction}

Ankle foot orthoses (AFOs) are external medical devices used to restore the natural gait pattern for patients with neurological and musculoskeletal disorders (Singer et al. 2014; Kobayashi et al. 2015; Ries et al. 2015; Choi et al. 2017). Depending on the design and the material, AFOs can be classified in different ways: currently, in the USA, the majority of the AFOs are custom fabricated (73\%) and made of thermoplastic materials (83\%) (American Board for Certification in Orthotics, Prosthetics \& Pedorthics, Inc 2015). Their production process is mostly time consuming and manual, where the craftsmen play a critical role (Mavroidis et al. 2011; International Committee of the Red Cross 2006). This way of manufacturing does not allow modification of the design parameters before the realization of the devices; therefore, it would be beneficial to quantify in advance the impact of AFO properties, such as stiffness and thickness, which are key factors for determining the amount of assistance the orthosis is able to provide (Bregman et al. 2012; Esposito et al. 2014; Kerkum et al. 2015). Currently, different research groups have been focusing on the use of $3 \mathrm{D}$ printing technologies, which enable high control of design characteristics: 3D printed AFOs are manufactured for healthy subjects and patients to study their contribution to the ankle biomechanics and/or compare their performance with the commonly prescribed AFOs (Mavroidis et al. 2011; Creylman et al. 2013; Arch and Stanhope 2015; Harper et al. 2014a, 2014b; Cha et al. 2017).

$3 \mathrm{D}$ printing technologies can be complemented with the construction of finite element (FE) models for patient-specific AFOs: they allow the assessment of the mechanical behavior and stress concentrations before manufacturing the devices and thus prevent wasted production time and errors (Chu and Reddy 1995; Syngellakis et al. 2000; Lee et al. 2006; Jamshidi et al. 2010). A more complete overview can be obtained if the FE results are coupled with dedicated experimental tests. This approach was used by different research groups for the mechanical evaluation of composite AFOs (Zou et al. 2014; Stier et al. 2015; Bellavita et al. 2017), thermoplastic AFOs (Zou et al. 2014), polycarbonate AFOs (Schrank et al. 2013) and AFOs realized in PA 12, glass-filled PA 12 and PA 11 (Faustini et al. 2008). However, none of these studies were simulating the devices during a continuous cycle 

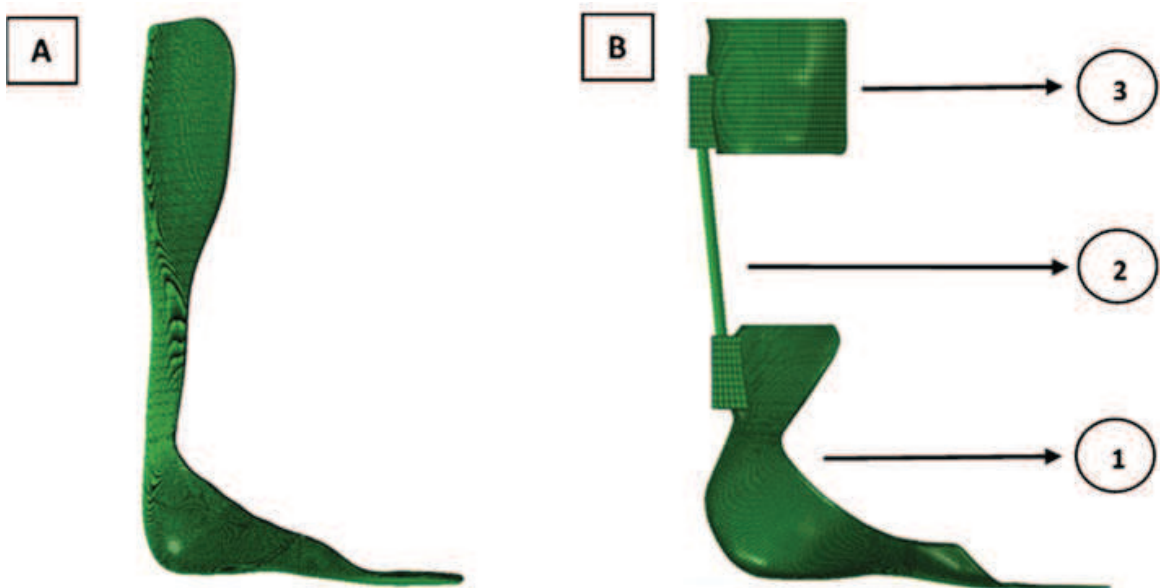

Figure 1. Virtual representation of the full shell (A) and the modular 3D printed AFOs (B): 1. foot part; 2. rods; 3. calf part.

of plantarflexion and dorsiflexion. Therefore, the possibility of virtually predicting the behavior of the AFOs for a complete range of motion, derived from the patients' gait assessment, could provide further information for the realization of the devices.

In this context, the present study proposes a new standardized framework for the creation of FE models of $3 \mathrm{D}$ printed patient-specific AFOs in order to quantify the ankle stiffness and the stress distribution during the second rocker of the gait. More specifically, the aim is the validation of these models through the utilization of a dedicated in-house developed mechanical testing rig (Ielapi et al. 2018), that establishes the AFO conditions replicated in the FE simulations. This represents an important step for the future application of the FE models in the prediction of the AFOs behavior in case of pathological conditions.

\section{Materials and methods}

In this study the mechanical properties of two different AFO designs are investigated: a full shell and a modular design. The full shell AFO consists of one entirely $3 \mathrm{D}$ printed part while the modular $\mathrm{AFO}$ is made of three parts: two fully 3D printed parts (foot and calf) connected by two carbon rods (Figure 1).

Using pyFormex software, an algorithm was developed to create a new regular mesh of the geometrical models of the AFOs provided in STL file format. The algorithm enables separate consideration of the internal and the external surfaces of the selected AFO and then slicing to obtain different polylines. Subsequently all the polylines are reconnected and a regular representation is obtained. The final mesh of the device is obtained by averaging the internal and external meshes. Quadrilateral elements were used for the construction of the modular design AFO mesh which has a uniform thickness. For the full shell mesh, which has a non uniform thickness, it was calculated by considering the distance between the final quadrilateral mesh and the initial STL. The final meshes ranged between 45000 and 67000 linear shell elements with reduced integration (S4R). A mesh sensitivity analysis performed over a range of 10 degrees in both dorsiflexion and plantarflexion, revealed that with these mesh numbers a converged solution was obtained. In fact, using different mesh grids (20500 S4R elements till 102500 S4R elements) revealed that the maximum difference in terms of stiffness is lower than $1 \%$ in both plantarflexion and dorsiflexion, while, in terms of stress distribution, equal to $0.6 \%$ in plantarflexion and $1.5 \%$ in dorsiflexion.

In this study, four patients (both children and adults) were selected and the pathologies considered were trauma, neuro-muscular disorder and cerebral palsy. Consequently, four patient-specific FE models were created: one model with the full shell design and three models with the modular design. The EU foot size of the full shell AFO was 40, defined as AFO A, while the modular AFOs, defined as AFO B, C and D, have 35,37 and $38 \mathrm{EU}$ foot size. For every modular AFO, the rods (thickness $=6 \mathrm{~mm}$ ) are made of carbon fiber reinforced polymer (CFRP). The foot and the calf part of the AFOs were realized in Polyamide 12 (PA 12). The full shell AFO, instead, is completely realized in PA 12 . Since this polymer owns complex visco-elasto-plastic properties, the virtual implementation was realized through a parallel rheological framework (PRF) model in the FE software Abaqus: the framework is based on the superposition of viscoelastic and elastoplastic networks in parallel, so to have an additive total stress response. 
Table 1. Nonlinear viscoelastic parameters for PA 12, where the stiffness ratio determines the contribution of each network and the parameters $q_{0}, n, m, a$, and $\dot{\varepsilon}_{0}$ are introduced for defining the creep behavior

\begin{tabular}{lcccccc}
\hline Network & Stiffness ratio & $\mathrm{q}_{0}\left[\mathrm{~N} / \mathrm{m}^{2}\right]$ & $n$ & $\mathrm{~m}$ & $\mathrm{a}$ & $\varepsilon_{0}^{*}[1 / \mathrm{s}]$ \\
\hline 1 & 0.162 & $2.52 \mathrm{E}+09$ & 1.081 & -0.026 & 0 & 1 \\
2 & 0.184 & 29.236 & 3 & -0.012 & 0 & 1 \\
\hline
\end{tabular}

Table 2. Plastic parameters for PA 12

\begin{tabular}{lc}
\hline Plastic stress [MPa] & Plastic strain \\
\hline 17.644 & 0.0 \\
34.096 & 0.005 \\
1.0 & 0.150 \\
\hline
\end{tabular}

Table 3. Hyperelastic parameters for PA 12

\begin{tabular}{lc}
\hline $\mathrm{C}_{10}[\mathrm{MPa}]$ & $\mathrm{D}_{1}[1 / \mathrm{MPa}]$ \\
\hline 395.986 & $6.335 \mathrm{e}-4$ \\
\hline
\end{tabular}

Table 4. Parameter for the carbon fiber reinforced polymer (CFRP) rods

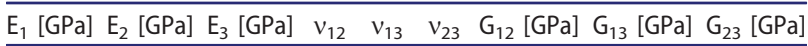
\begin{tabular}{lllllllll}
\hline 95 & 8 & 8 & 0.35 & 0.35 & 0.35 & 2 & 2 & 3 \\
\hline
\end{tabular}

In particular, the framework is intended for polymers and elastomeric materials which exhibit a nonlinear viscous behavior, implying hysteresis effects and undergoing large deformations (Hurtado et al. 2013; Lapczyk and Hurtado 2014), which makes it suitable for PA 12. The nonlinear viscous effects were modeled using the power law model formulation, while the plasticity was expressed with the stress values at the corresponding plastic strain (Tables 1 and 2). The elastic response was specified using hyperelastic neohookean material coefficients (Table 3). All the parameters were derived from experimental tests carried out on samples of the material (Lammens et al. 2017) and they are suitable for describing the behavior of PA 12 during a static analysis. The material properties of the CFRP rods were also obtained experimentally (Table 4).

The loads and boundary conditions used in the FE models correspond to a dedicated experimental setup (Figure $2 \mathrm{~A}$ and $\mathrm{B}$ ) specifically designed for the evaluation of the ankle stiffness of AFOs during the second rocker of the gait (Ielapi et al. 2018). The fixation of the AFOs in the device is via a custom made clamping system which makes use of medium density fiberboard (MDF) replica blocks of the patients' leg, which contain anatomical landmarks for allowing the alignment of the test rig axis with the anatomical ankle axis. The loads applied to the FE models are the dorsiflexion and the plantarflexion, respectively defined as the movement of the AFO calf towards the foot section in the sagittal plane and the reverse movement. The ranges of motion for each AFO are derived from the gait assessment of the patients when they walked with their AFO (Table 5).

The rotation is applied around a point identified as the virtual ankle joint, which is the midpoint of the anatomical lateral and medial ankle malleoli, recorded during the gait assessment of the patient. Knowing the location of the virtual ankle joint, allows calculation of the moments acting on the device during the rotation. In this way it is possible to define the AFO stiffness, which is the moment around the ankle joint exerted by the AFO per degree of ankle joint rotation (Bregman et al. 2009). The evaluation of the AFO ankle stiffness is performed using four values, corresponding to the four zones of the angle vs torque graph: Plantarflexion Loading (PL), Plantarflexion Unloading (PU), Dorsiflexion Loading (DL) and Dorsiflexion Unloading (DU). For each of these zones, the stiffness value is quantified as the slope of the angle vs torque curve in the specific quadrant.

Concerning the boundary conditions, depending on the height of the MDF blocks employed, the AFO foot region is kept fixed in all the directions using an encastre constraint, thus to represent the AFO behavior during the second rocker of the gait. The internal surface of the modular calf part or the upper part of the full shell design is constrained to follow the movement of a reference point by a kinematic coupling, which induces the deformation of the device and represents the connection of the AFO to the leg of the patient. This point is derived from the anatomical landmarks of the patients' knee and is positioned in correspondence to the AFOs straps in order to be aligned with the shank axis (Figure $2 \mathrm{~B}$ ). The calf reference point and the virtual ankle joint are then linked by a "Slot + Revolute" connector which allows the calf point to slide up and down along and to rotate around the shank axis with respect to the virtual ankle joint, mimicking the AFO behavior in the test rig, where the motor induces the movement of the U-shaped frame around the ankle rotation axis of the setup.

As stated above, the PRF model is able to reproduce the hysteresis effects of the material given by the loading-unloading of the devices during plantarflexion and dorsiflexion, which contribute to part of the total hysteresis. In fact, there is another contribution to the energy dissipation induced by the interaction of the AFO with the test rig (i.e. the contact between the MDF blocks and the AFO) and between the components of the test rig itself. Mimicking these factors in 


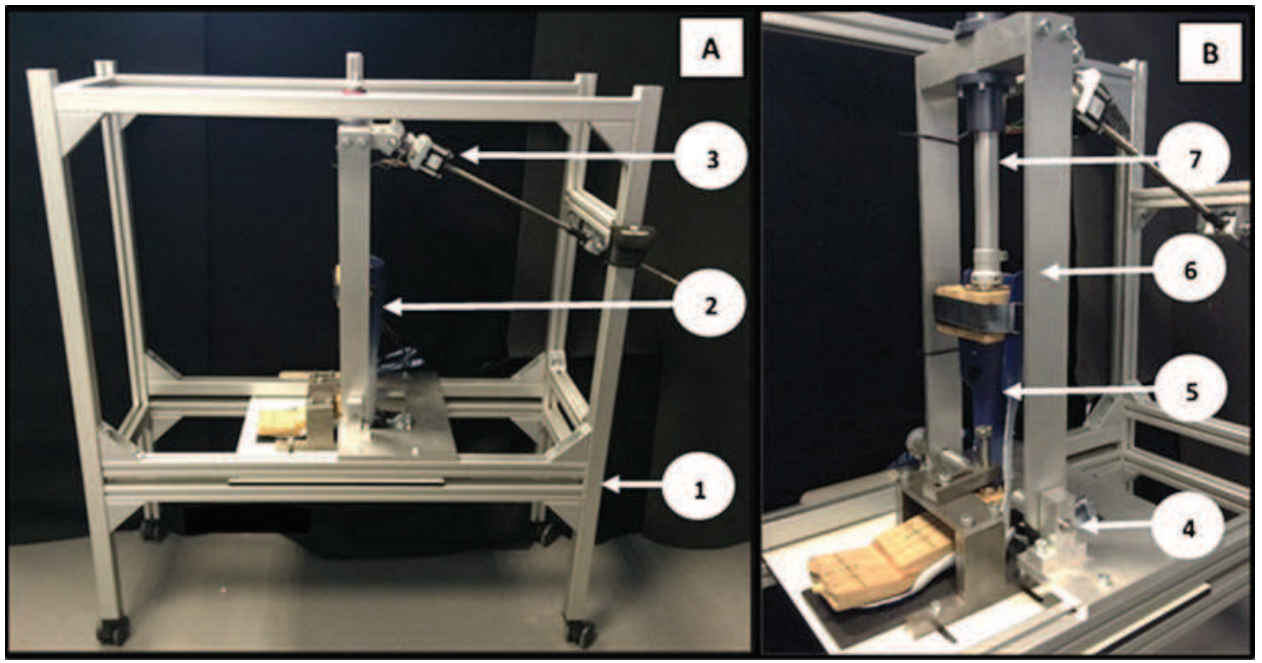

Figure 2. A and B: Overview of the experimental setup for testing the $3 \mathrm{D}$ printed AFOs: 1. External frame; 2. AFO; 3 . Linear Motor; 4. Ankle rotation axis; 5 . Closer view of the clamped AFO; 6 . U-shaped frame; 7. Shank axis.

a standardized manner is possible by specifying a friction coefficient for the connector along the sliding direction of the shank axis. Furthermore, by specifying a damping coefficient for the connector allows representation of the forces resulting from the AFO straps around the calf part. This represents an artificial parameter used to describe the AFO conditions when the angular deflection is equal to zero. The values of the friction and damping coefficients, 0.3 and $30 \mathrm{~N} / \mathrm{mm} / \mathrm{s}$ respectively, are the same for each AFO, in order to obtain a predictive and standardized FE framework applicable to each patient-specific AFO tested in the experimental setup.

Because of the available equipment, the experimental results were recorded with a sampling rate of $10 \mathrm{~Hz}$ at a velocity of 1 degree/s and then filtered with a $4^{\text {th }}$ order low pass Butterworth filter with a cut-off frequency at $0.2 \mathrm{~Hz}$. The FE analysis was performed using Abaqus/Standard 2017. The outcomes in terms of moments and rotational angles are then used to calculate the ankle stiffness of each patient-specific AFO and compared with the experimental results.

\section{Results}

In this section the experimental and computational results are reported.

Figure 3 depicts an overall good correlation for all the tested AFOs. All the curves show the presence of hysteresis and nonlinearities in the unloading phases. It is also visible that all the devices behave differently in dorsiflexion and plantarflexion. This is confirmed by analyzing the ankle stiffness values for each quadrant of the graph, summarized in Table 6 .
Table 5. AFO's ranges of motion

\begin{tabular}{lcc}
\hline AFO & Plantarflexion $\left[^{\circ}\right]$ & Dorsiflexion $\left[^{\circ}\right]$ \\
\hline A & -2 & 3 \\
B & -6 & 3 \\
C & -5 & 5 \\
D & -8 & 8 \\
\hline
\end{tabular}

Plantarflexion is indicated with negative angles and dorsiflexion with positive angles.

In Table 7, the absolute and relative errors are reported. These values confirm the overall good agreement noticed in Figure 3; in plantarflexion, the maximum error is reached by the $\mathrm{AFO} C(8.45 \%)$ and $\mathrm{D}$ (10.66\%), while in dorsiflexion by the AFO A (10.14\%) and C (10.38\%), respectively in loading and unloading.

Figure 4 shows the von Mises stress distribution for the AFOs during dorsiflexion and plantarflexion. The full shell AFO is characterized by intermediate stress concentrations around the trimlines, while for the modular AFOs, the stress concentrations occur around the blocks on the foot part. In dorsiflexion, the highest values of stress are reached by the AFO C $(47.35 \mathrm{MPa})$ and D (46.57 MPa), while, in plantarflexion by the AFO B (48.33 MPa). Concerning the CFRP rods, the study of the stress in the fiber direction showed lower values than the parts in PA 12. In general, the values shown by AFO B, C and D are higher than the ultimate strength value (equal to $34.096 \mathrm{MPa}$ ) indicated for PA 12 in Table 2, which might lead to the failure of the devices in the reality (Deckers et al. 2018).

\section{Discussion}

This study presents a novel dedicated framework, combining experimental and numerical methods, to 


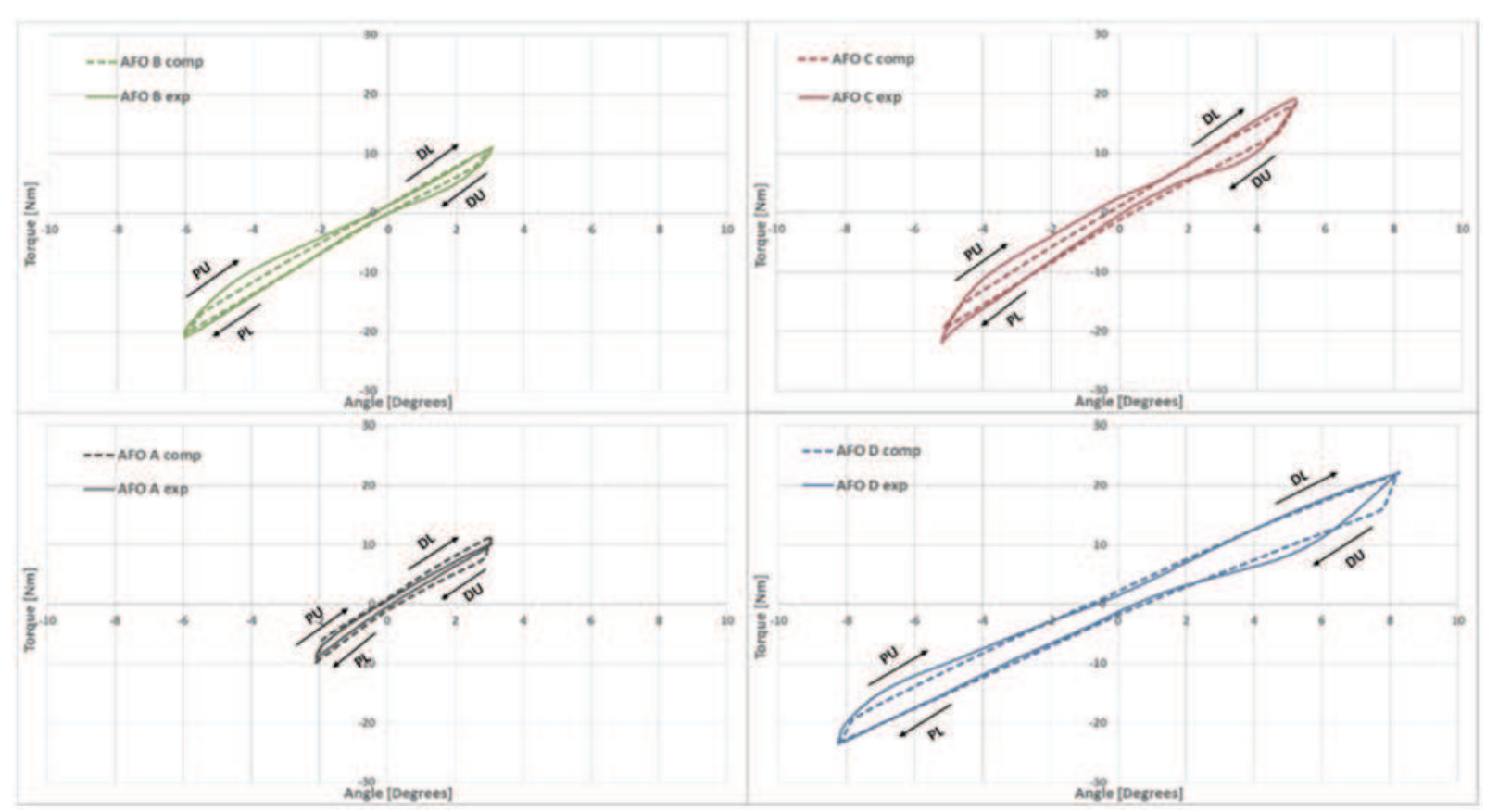

Figure 3. Comparison between the experimental (exp) and computational (comp) stiffness curves for the four AFOs during Plantarflexion Loading (PL), Plantarflexion Unloading (PU), Dorsiflexion Loading (DL) and Dorsiflexion Unloading (DU). The ranges of motion used for each AFO are contained in Table 5.

Table 6. Comparison between experimental and computational ankle stiffness results of the four AFOs during Plantarflexion Loading (PL), Plantarflexion Unloading (PU), Dorsiflexion Loading (DL) and Dorsiflexion Unloading (DU). The ranges of motion used for each AFO are contained in Table 5

\begin{tabular}{|c|c|c|c|c|c|c|c|c|}
\hline \multirow[b]{2}{*}{ AFO } & \multicolumn{2}{|c|}{$\mathrm{PL}$} & \multicolumn{2}{|c|}{ PU } & \multicolumn{2}{|c|}{$\mathrm{DL}$} & \multicolumn{2}{|c|}{ DU } \\
\hline & Exp. $\left[\mathrm{Nm} /{ }^{\circ}\right]$ & Comp. $\left[\mathrm{Nm} /{ }^{\circ}\right]$ & Exp. $\left[\mathrm{Nm} /{ }^{\circ}\right]$ & Comp. $\left[\mathrm{Nm} /{ }^{\circ}\right]$ & Exp. $\left[\mathrm{Nm} /{ }^{\circ}\right]$ & Comp. $\left[\mathrm{Nm} /{ }^{\circ}\right]$ & Exp. $\left[\mathrm{Nm} /{ }^{\circ}\right]$ & Comp. $\left[\mathrm{Nm} /{ }^{\circ}\right]$ \\
\hline $\bar{A}$ & 4.00 & 4.13 & 4.00 & 3.92 & 3.23 & 3.56 & 3.48 & 3.27 \\
\hline B & 3.42 & 3.40 & 3.08 & 3.34 & 3.15 & 3.21 & 2.97 & 3.28 \\
\hline C & 3.94 & 3.60 & 3.69 & 3.68 & 3.21 & 3.42 & 3.01 & 3.32 \\
\hline D & 2.69 & 2.62 & 2.42 & 2.68 & 2.64 & 2.50 & 2.41 & 2.38 \\
\hline
\end{tabular}

Table 7. Absolute and relative errors for the AFOs ankle stiffness of the four patients during Plantarflexion Loading (PL), Plantarflexion Unloading (PU), Dorsiflexion Loading (DL) and Dorsiflexion Unloading (DU). The ranges of motion used for each AFO are contained in Table 5

\begin{tabular}{|c|c|c|c|c|c|c|c|c|}
\hline \multirow[b]{2}{*}{$\mathrm{AFO}$} & \multicolumn{2}{|c|}{$\mathrm{PL}$} & \multicolumn{2}{|c|}{ PU } & \multicolumn{2}{|c|}{$\mathrm{DL}$} & \multicolumn{2}{|c|}{ DU } \\
\hline & Abs. $\operatorname{Err}\left[\mathrm{Nm} /{ }^{\circ}\right]$ & Rel. Err. [\%] & Abs. $\operatorname{Err}\left[\mathrm{Nm} /{ }^{\circ}\right]$ & Rel. Err. [\%] & Abs. $\operatorname{Err}\left[\mathrm{Nm} /{ }^{\circ}\right]$ & Rel. Err. [\%] & Abs. $\operatorname{Err}\left[\mathrm{Nm} /{ }^{\circ}\right]$ & Rel. Err. [\%] \\
\hline A & 0.13 & 3.14 & 0.08 & 2.04 & 0.33 & 10.14 & 0.21 & 6.14 \\
\hline B & 0.02 & 0.61 & 0.26 & 8.58 & 0.07 & $\overline{2.11}$ & 0.31 & 10.30 \\
\hline C & 0.33 & 8.45 & 0.01 & 0.36 & 0.21 & 6.61 & 0.31 & 10.38 \\
\hline D & 0.10 & $\overline{2.31}$ & 0.26 & 10.66 & 0.14 & 5.42 & 0.03 & $\overline{1.33}$ \\
\hline
\end{tabular}

evaluate the ankle stiffness and the stress distribution of AFOs during the second rocker of the gait, through the use of an advanced material model for the PA 12, which was never used in the past for these devices. The rationale behind this is to further create a scientifically robust method to design and manufacture AFOs with specific mechanical properties in a reliable and repetitive way. To date, most AFOs are still crafted manually (International Committee of the Red Cross 2006; Mavroidis et al. 2011). By using 3D printing technology, it would be easier to prevent the systematic errors and guarantee shape and functional characteristics that were initially established in the original CAD models of the AFOs (Schrank and Stanhope 2011).

The experimental results described above focus on the evaluation of the stiffness around an axis aligned to the anatomical ankle joint over a patient-specific range of motion in dorsiflexion and plantarflexion, chosen according to the data coming from the gait 


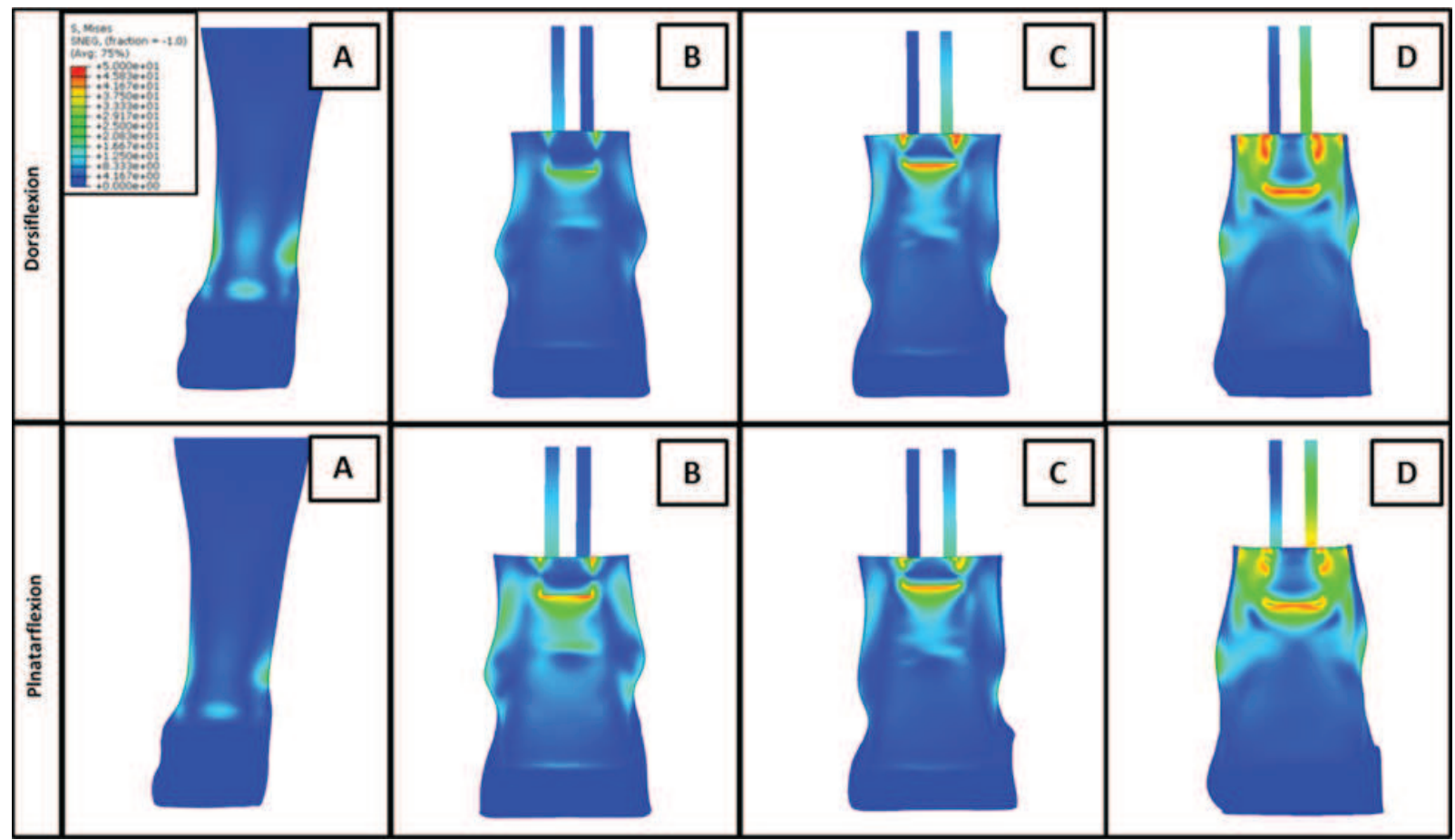

Figure 4. Von Mises stress distribution on the AFO A, B, C and D at the maximal dorsiflexion and plantarflexion.

analysis in order to ensure the best approximation of the AFO stiffness felt by the patient during gait. The developed modelling strategy gives a fast indication of the AFO ankle stiffness and stress distribution. Dedicated algorithms were developed in pyFormex to convert STL files describing the AFO geometry and used for 3D printing into high quality finite element models. Solving the numerical problem with Abaqus required 30 minutes on average. The models show overall good agreement with the experimental data (Figure 3). Four different ankle stiffness values are considered for each quadrant of the angle vs. torque curve, as most of the patient-specific AFOs show a different behavior in plantarflexion compared to dorsiflexion due to their shape (Table 6). The magnitude of these stiffness values is in line with that used in other research studies for investigating 3D printed AFOs (Schrank et al. 2013; Choi et al. 2017). AFO A seems to be the stiffest in plantarflexion whereas in dorsiflexion, $\mathrm{AFO} \mathrm{A}, \mathrm{B}$ and $\mathrm{C}$ have similar values. AFO D is the most flexible AFO in both dorsiflexion and plantarflexion. The visual agreement is confirmed by the data contained in Table 7 , which show the ability of the FE models to predict their mechanical behavior. This was achievable through the utilization of an advanced material model for the PA 12 in combination with the boundary conditions of the experimental setup, mimicking as closely as possible the behavior of the 3D printed AFOs. All the stiffness plots showed the presence of hysteresis: the hysteresis area represents the dissipated energy as heat during the deformation (loading) and the recovery phases (unloading). This is dependent upon the strain rate employed to deform the devices (ÖZkaya and Nordin 1999) constructed from PA-12, which has viscoelasto-plastic properties (Lammens et al. 2017), and by the friction present between the AFO and the test rig and between the components of the test rig itself. For this reason both friction and damping coefficients were used. As the focus of the current study was to derive the ankle stiffness values, friction was not calculated experimentally but chosen by curve fitting. The damping coefficient instead is an artificial parameter, which has no testing equivalent, used to represents the AFO conditions when the angular deflection is equal to zero. By using one friction and damping coefficient for all the AFOs allow standardization of the computational framework, where the prediction is only depending on the shape and geometry of the patient-specific devices. The presence of hysteresis due to friction and the nonlinear behavior of the devices affects most of the stiffness values in the unloading phases (see Figure 3), which might cause underestimations of the calculated stiffness values.

In Figure 4 the von Mises stress distribution for the four AFOs is depicted. For the AFO A, which has a full shell design, the highest stresses are concentrated around the trimlines, while for the modular 
AFOs, the highest stresses are concentrated around the blocks on the foot part that are connected to the CFRP rods. Since this is a preliminary analysis, further tests are needed to confirm if these stress values will cause the failure of the devices (Deckers et al. 2018). For evaluating the stress on the rods made in CFRP, which is stiffer (Table 4) and has a failure stress higher than PA 12 (Lammens 2015), the stress in the fiber direction was studied. This showed stress values lower than the parts in PA 12. Deckers et al. showed that failure might occur if inaccurate cutting and grinding of the rods is applied, which can alter their behavior.

The FE analysis performed in this study takes into account only one loading cycle for each patient-specific AFO. The material model, in fact, is able to mimic the behavior of the devices during a static analysis, but it is not designed to predict the response of the devices during fatigue. In order to further enhance the accuracy of the model (i.e. the nonlinearities during unloading), the straps used to fix the patient leg on the AFO or the MDF blocks could have been modeled; this would increase the complexity of the model (i.e. material model for the straps, contact properties between AFO and blocks etc.) and decrease the (time) efficiency. In addition, the use of dynamic friction and damping coefficients would decrease the percentage errors but, at the same time, make the framework less predictive and standardized if one would calibrate these values for each AFO individually. Because of the available instrumentation within the setup, it was only possible to perform tests at a speed of $1 \%$; in the future, the use of a new equipment will allow the comparison for speed values close to those seen during gait.

In conclusion we can say that the use of advanced computer modelling algorithms together with 3D printing techniques is a strong combination acting to improve the manufacturing process of the AFOs and represents a big step for the future prediction of the AFOs behavior in case of pathological conditions.

\section{Declaration of interest statement}

The authors declare no conflicts of interest.

\section{Funding}

This research was supported by VLAIO (Flanders Innovation \& Entrepreneurship) and the A_STREAM_AFO project (Applied Structural Engineering of AM Materials for Ankle Foot Orthosis; project numbers: 140164 \& 140165) under the SIM (Strategic Initiative Materials in
Flanders) research program STREAM (STRuctural Engineering materials through Additive Manufacturing).

\section{References}

American Board for Certification in Orthotics, Prosthetics \& Pedorthics, Inc. 2015. Practice analysis of Certified Practitioners in the Disciplines of Orthotics and Prosthetics.

Arch ES, Stanhope SJ. 2015. Passive-dynamic ankle-foot orthoses substitute for ankle strength while causing adaptive gait strategies: a feasibility study. Ann Biomed Eng. 43(2):442-450.

Bellavita G, Cocconcelli M, Castagnetti D, Rubini R. 2017. Development and validation of a numerical model for the optimization of a brace for lower limb. In: Silva L, editor. Materials design and applications. Advanced structured materials, vol 65. Cham: Springer.

Bregman DJJ, Harlaar J, Meskers CG, de Groot V. 2012. Spring-like Ankle Foot Orthoses reduce the energy cost of walking by taking over ankle work. Gait Posture. 35(1):148-153.

Bregman DJJ, Rozumalski A, Koops D, de Groot V, Schwartz M, Harlaar J. 2009. A new method for evaluating ankle foot orthosis characteristics: BRUCE. Gait \& Posture. 30:144-149.

Cha YH, Lee KH, Ryu HJ, Joo IW, Seo A, Kim DH, Kim SJ. 2017. Ankle-foot orthosis made by 3D printing technique and automated design software. Appl Bionics Biomech. 2017:9610468.

Choi H, Peters KM, MacConnell M, Ly K, Eckert E, Steele KM. 2017. Impact of ankle foot orthosis stiffness on Achilles tendon and gastrocnemius function during unimpaired gait. J Biomech. 64:145-152.

Chu T, Reddy NP. 1995. Stress distribution in the anklefoot orthosis used to correct pathological gait. J Rehabil Res Dev. 32(4):349-360.

Creylman V, Muraru L, Pallari J, Vertommen H, Peeraer L. 2013. Gait assessment during the initial fitting of customized selective laser sintering ankle foot orthoses in subjects with drop foot. Prosthet Orthot Int. 37(2): 132-138.

Deckers JP, Vermandel M, Geldhof J, Vasiliauskaite E, Forward M, Plasschaert F. 2018. Development and clinical evaluation of laser sintered ankle foot orthoses. Plast Rubber Compos. 47(1):42-46.

Esposito ER, Blanck RV, Harper NG, Hsu JR, Wilken JM. 2014. How does ankle-foot orthosis stiffness affect gait in patients with lower limb salvage? Clin Orthop Relat Res. 472(10):3026-3035.

Faustini MC, Neptune RR, Crawford RH, Stanhope SJ. 2008. Manufacture of passive dynamic ankle-foot orthoses using selective laser sintering. IEEE Trans Biomed Eng. 55(2):784-790.

Harper NG, Russell EM, Wilken JM, Neptune RR. 2014a. Selective laser sintered versus carbon fiber passivedynamic ankle-foot orthoses: a comparison of patient walking performance. J Biomech Eng. 136(9):091001

Harper NG, Russell EM, Wilken JM, Neptune RR. 2014b. The influence of ankle-foot orthosis stiffness on walking 
performance in individuals with lower-limb impairments. Clin Biomech (Bristol, Avon). 29(8):877-884.

Hurtado JA, Lapczyk I, Govindarajan SM. 2013. Parallel rheological framework to model non-linear viscoelasticity, permanent set, and mullins effect in elastomers. Constitutive Models for Rubber. VIII:95-100.

Ielapi A, Vasiliauskaite E, Hendrickx M, Forward M, Lammens N, Van Paepegem W, Deckers JP, Vermandel M, De Beule M. 2018. A novel experimental setup for evaluating the stiffness of ankle foot orthoses. BMC Res Notes. 11(1):649.

International Committee of the Red Cross. 2006. Anklefoot orthosis: manufacturing guidelines.

Jamshidi N, Hanife H, Rostami M, Najarian S, Menhaj MB, Saadatnia M, Salami F. 2010. Modelling the interaction of ankle-foot orthosis and foot by finite element methods to design an optimized sole in steppage gait. J Med Eng Technol. 34(2):116-123.

Kerkum YL, Buizer AI, van den Noort JC, Becher JG, Harlaar J, Brehm M. 2015. The effects of varying ankle foot orthosis stiffness on gait in children with spastic cerebral palsy who walk with excessive knee flexion. PLoS ONE. 10(11):e0142878.

Kobayashi T, Singer ML, Orendurff MS, Gao F, Daly WK, Foreman KB. 2015. The effect of changing plantarflexion resistive moment of an articulated ankle-foot orthosis on ankle and knee joint angles and moments while walking in patients post stroke. Clin Biomech (Bristol, Avon). 30(8):775-780.

Lammens N, Kersemans M, De Baere I, Van Paepegem W. 2017. On the visco-elasto-plastic response of additively manufactured polyamide-12 (pa-12) through selective laser sintering. Polymer Testing. 57:149-155.

Lammens N. 2015. Optimization of Optical Fiber Coating and Interrogator Geometry for Embedding in Composite Laminates. [dissertation]. Gent (BE): Gent University.

Lapczyk I, Hurtado J. 2014. A viscoelastic-elastoplastic finite strain framework for modeling polymers. ASME International Mechanical Engineering Congress and Exposition, Volume 9: Mechanics of Solids, Structures and Fluids ():V009T12A096. doi:10.1115/IMECE2014-36831.
Lee Y, Choi Y, Kim H, Lee H, Cho L. 2006. A study on the structural stress analysis of plastic ankle foot orthosis (AFO) under dorsiflexion and plantarflexion conditions. Int J Mod Phys B. 20(25n27):4559-4564.

Mavroidis C, Ranky RG, Sivak ML, Patritti BL, DiPisa J, Caddle A, Gilhooly K, Govoni L, Sivak S, Lancia M, et al. 2011. Patient specific ankle-foot orthoses using rapid prototyping. J Neuro Eng Rehab. 8:1.

ÖZkaya N, Nordin M. 1999. Fundamentals of biomechanics: equilibrium, motion, and deformation. New York: Springer.

Ries AJ, Novacheck TF, Schwartz MH. 2015. The efficacy of ankle-foot orthoses on improving the gait of children with diplegic cerebral palsy: A multiple outcome analysis. PM R. 7(9):922-929.

Schrank ES, Hitch L, Wallace K, Moore R, Stanhope SJ. 2013. Assessment of a virtual functional prototyping process for the rapid manufacture of passive-dynamic anklefoot orthoses. J Biomech Eng. 135(10):101011-101017.

Schrank ES, Stanhope SJ. 2011. Dimensional accuracy of ankle-foot orthoses constructed by rapid customization and manufacturing framework. JRRD. 48(1):31-42.

Singer ML, Kobayashi T, Lincoln LS, Orendur MS, Foreman KB. 2014. The effect of ankle-foot orthosis plantarflexion stiffness on ankle and knee joint kinematics and kinetics during first and second rockers of gait in individuals with stroke. Clin Biomech. 29(9):1077-1080.

Stier B, Simon J, Reese S. 2015. Numerical and experimental investigation of the structural behavior of a carbon fiber reinforced ankle-foot orthosis. Med Eng Phys. 37(5):505-511.

Syngellakis S, Arnold MA, Rassoulian H. 2000. Assessment of non-linear behavior of plastic ankle foot orthoses by the finite element method. Proc Inst Mech Eng $\mathrm{H}$. 214(5):527-539.

Zou D, He T, Dailey M, Smith KE, Silva MJ, Sinacore DR, Mueller MJ, Hastings MK. 2014. Experimental and computational analysis of composite ankle-foot orthosis. J Rehabil Res Dev. 51(10):1525-1536. 